\title{
Salivary Diagnosis: A Single Drop Can Diagnose Many
}

\author{
Neeraj Arora ${ }^{1}$, Manpreet S Walia ${ }^{2}$, Mamta Malik ${ }^{3}$, Ravinder Singh Saini ${ }^{4}$, \\ Saryu Arora ${ }^{5}$ and Sanjeev Laller ${ }^{6}$ \\ ${ }^{1}$ Department of Microbiology, Government hospital, Panchkula, Haryana \\ ${ }^{2}$ Department of Prosthodontics, H.S. Judge Institute of Dental Sciences and Hospital, \\ Chandigarh, India \\ ${ }^{3,6}$ Department of Oral Medicine and Radiology ,PDM Dental College and Research Institute, \\ Bhadurgarh \\ ${ }^{4}$ Department of Prosthodontics, Desh Bhagat Dental College,Muktsar, Punjab \\ ${ }^{5}$ Department of Prosthodontics, Swami Devi Dayal Hospital and Dental College, Barwala
}

Correspondence should be addressed to: Neeraj Arora; neerajaurora76@gmail.com

Received date: 3 December 2013; Accepted date: 8 September 2014;

Published date: 3 January 2016

Academic Editor: Ebru Emekli-Alturfan

Copyright @ 2016. Neeraj Arora, Manpreet S Walia, Mamta Malik, Ravinder Singh Saini, Saryu Arora and Sanjeev Laller. Distributed under Creative Commons CC-BY 4.0

\begin{abstract}
The oral cavity has a moist environment and a film of fluid, the fluid is saliva which comprises of mixture of secretions from major and minor salivary glands. Saliva provides an easily available, now increasing diagnostic medium for a rapidly, widening range of diseases and clinical situations. It is used in the diagnosis of various oral and systemic diseases. Within the past 20 years, more than 2,500 citations have focused on the diagnostic value of the oral fluids particularly saliva. This paper highlights the role of saliva in the diagnosis of various diseases and disorders.
\end{abstract}

Keywords: saliva, diagnosis, systemic diseases.

\section{Introduction}

Saliva is a watery and usually frothy substance which is an ultra filtrate of the blood, produced in the mouths of humans and most of other animals. Advances in the use of saliva as a diagnostic fluid have been tremendously affected by current technological developments. Saliva is used not only to monitor the level of internal 
molecules in the body including polypeptide and steroids hormones and antibodies but Saliva is also used to monitor the level of certain chemicals like alcohol and drugs in the body. Salivary fluid is used and aids in the diagnosis of measles, mumps, rubella, hepatitis $\mathrm{A}, \mathrm{B}$ and $\mathrm{C}$ and HIV - 1 and 2, sarcoidosis, tuberculosis, lymphomas and Sjogren's syndrome etc manifested systemically and orally. ${ }^{1}$

\section{Need for salivary diagnosis}

Saliva has clinical advantages over other body fluids like blood, serum/urine in the diagnosis. It is easy to collect, store and shift. Patient can collect sample at home when clinically relevant or in other places, including the workplace, where collecting blood or urine may be difficult. It is obtained at low cost and in sufficient quantities for analysis. Its non invasive collection technique reduces anxiety in subjects and is safer for health care workers than serum analysis with its associated exposure to needles and possibly AIDS or Hepatitis viruses. Most molecules found in blood and urine are found in saliva, although the concentrations are one tenth to one thousandth of those in blood. ${ }^{1}$

\section{Saliva in oral diseases}

To start with the most common oral diseases diagnosed by saliva are dental caries and periodontal diseases. Saliva can be used to assess susceptibility to dental caries and to detect oral, fungal and periodontal infections.

\section{Diagnosis of dental caries}

Saliva is important for the health of both the oral soft and the hard tissue. It influences the tooth structure by affecting the caries process. Individual components of saliva are related in some way or the other to dental caries. Physical, chemical and biological properties of saliva may influence the susceptibility to caries which are beneficial in the diagnosis of caries. Physical factors influencing dental caries are: Salivary flow, Salivary $\mathrm{pH}$ : $\mathrm{pH}$ at which any particular saliva ceases to be supersaturated with $\mathrm{Ca}++$ and phosphate ions is called Critical $\mathrm{pH}=5.5$. The $\mathrm{pH}$ falls below critical $\mathrm{pH}$ when there is acid production by plaque microflora. This acid is partly neutralized by carbonate in saliva and salivary volume as it decreases during sleep hours predisposing to caries. Biological factors influencing dental caries are lysozyme. They attack bacteria and aid thiocyanate ions in entering the bacteria where these become bactericidal. Ig antibodies can destroy oral bacteria causing dental caries and finally leukocytes i.e. PMNS are predominant in caries prone population. Chemical factors influencing dental caries are buffering capacity in which salivary buffers neutralize the acids produced by bacterial flora. Buffering agents present in saliva are bicarbonates, phosphates, urea and ammonia ${ }^{2,3}$.

Saliva in the diagnosis of BABY BOTTLE tooth decay ${ }^{4}$.

It was demonstrated that- Babies sleep more than adults and less saliva is produced during sleep. Infant's salivary antibody titre is very low and in addition to this Salivary flow in vicinity to the maxillary anterior teeth is noticeably low and slow because of gravity and the location of the glandular ducts. Also, sucking action during nursing puts resistance to salivary flow and, to that stage infant lip muscles are not well and sufficiently developed to remain "sealed" and as a result of which saliva on the upper incisors tends to evaporate and the incisors become dry. In infant's mouth, there is little mixing of saliva with infant's saliva containing only half the concentration of secretory IgA as compared to the one found in adult's saliva. All the above mentioned factors associated with abundance of streptococcus mutans due to frequent feeding and the availability of sugars in bottle milk/lactose in mother milk as substrate for bacteria result in nursing bottle caries ${ }^{4}$.

\section{Diagnosis of periodontal diseases}

As saliva contains locally and systemically derived periodontal disease markers and salivary sample can be easily collected, this 
property of saliva offers the basis for a patient specific diagnostic test for periodontitis. Within minutes after an oral prophylaxis, salivary glycoproteins begin to adsorb onto the tooth surfaces, forming an amorphous coating on the teeth called as acquired pellicle. Salivary pellicles not only form on tooth surfaces but also mucosal epithelium, dental appliances, restoration and orthodontic appliances. The association and interaction between micro organisms and salivary components in the pellicles affects first microbial adherence and is recognized as a primary step of plaque formation and associated disease. Cystatins acidic proline rich proteins, $\alpha$ amylase, secretory IgA and MG2 are the commonly found salivary proteins and in the pellicles formed on orthodontic appliances. All these factors act as receptors for bacterial adhesion of organisms like S mutans and S.gordonii to orthodontic brackets enhancing susceptibilities to enamel white spot formation. S.sanguis and A.viscosus are the pioneer microbes which colonize the tooth surface by adhering to salivary glycoproteins. After the initial colonization phase, a fairly growth occurs by colonization of streptococci perpendicular to the tooth surface followed by Actinomyces and Veillonella, subsequently by filamentous Nocardia and Bacterionema. These invasion changes the plaque environment by decreasing the $\mathrm{pH}$ and in- oxygen tension, which allow anaerobic organism like spirochetes to enter the plaque. Finally the supra gingival plaque community reaches maturity. The organisms that colonize the early plaque within the first 24 hours are generally well tolerated by the host, but the mature plaque has the potential to cause both dental caries and gingivitis, which allows organisms to enter the sub- gingival environment if left untreated to cause periodontitis. Chemical composition of salivary pellicle is similar to saliva containing proline rich proteins, mucins, blood group antigens, Igs, albumin, lysozyme, amylase, cysteine containing phosphoproteins, IgA, lactoferrins \& statherins. Proline rich proteins, IgA, lactoferrin, lysozyme and amylase may bind specifically to plaque bacteria and act as receptors for attachment. Composition levels similar to salivary pellicle can be monitored to reach the periodontal disease diagnosis ${ }^{5,6}$.

\section{Saliva in the diagnosis of oral infections}

With new and very sensitive techniques, the low levels of antibody in oral fluids relative to serum is not a limitation, and the safety, ease and low cost of collection are major benefits in large scale studies. The sensitivity of technologies currently available has marketedly increased to where the lack of saliva or other forms of trickle down salivation can be used to monitor numerous diseases and clinical concerns. Dentists can look forward to a new era in which oral fluid can be seen as a valuable friend rather than as enemy and tests in dental office will become common place ${ }^{7}$.

Examples of molecularly
$\begin{aligned} & \text { determinants used in saliva } \\ & \text { diagnostics }\end{aligned}{ }^{7,9}$ :

Detecting salivary bacterial organism most commonly Streptococcus mutans and Lactobacillus acidophilus, both responsible for the common dental problem called dental caries, and $P$. gingivalis which counts as a causative factor for periodontal disease

Detecting viruses using antibodies which are specific for a viral antigen like-Measles, Mumps, Rubella, Hepatitis A \& B and HIV 1 $\& 2$

Detection of microbe-specific antigenic determinants like CA-15 epidermal growth factor which is proposed breast cancer biomarkers and Neuraminidase, an enzyme associated with influenza A and B infection. And finally Glutamic acid decarboxylase autoantibody which is proposed predictive biomarker for most common immune disease -type I diabetes.

\section{Saliva in systemic diseases}

As it is said that oral cavity is the mirror of systemic diseases, physicians/clinicians are showing interest in using salivary 
analytical method to diagnose systemic disease and to monitor general health. The reason behind it is the ability of newer and latest diagnostic tools such as: sensitive enzyme linked immunosorbent assays and technologies to distinguish a range of salivary components which are biomarkers for changes in the overall general health. $10,11,12$

\section{Respiratory Diseases}

Salivary enzymes from fingival sulcus result in the removal of fibronectin by exposure to proteases which unmask mucosal surface adhesion receptors for respiratory pathogen adhesives. Hence, in subjects having periodontal disease and elevated levels of proteolytic bacteria like $P$. gingivalis and spirochaetes, protease activity alters the mucosal epithelium to increase the adhesion and colonization of respiratory pathogens. The best example is the chronic respiratory infection especially in children which is often associated with specific secretory IgA deficiency ${ }^{10}$

\section{Diabetes}

Literature had shown that blood glucose concentrations were determined in nondiabetic healthy individuals and patients with diabetes mellitus-type II. But, salivary glucose was only found in the saliva of patients with diabetes mellitus, while the salivary samples of healthy subjects did not show the presence of glucose. A significant correlation was found between salivary and blood concentrations in the diabetic samples. Thus, saliva can be used as a diagnostic tool for the diagnosis of diabetes in place of painful prick during blood glucose estimation ${ }^{11}$

\section{Chronic Heart Failure}

Salivary endothelin-1 concentration is elevated in patients with chronic heart failure and can be used to assess disease severity. Plasma concentrations of the vasoconstrictor peptide Endothelin-1 are raised 2-3 fold in patients with chronic heart failure (CHF). Endothelin - 1 concentration in saliva are substantially higher than those in plasma ${ }^{12}$.

\section{Cushing's syndrome and Addison's disease}

The level of cortisol content in saliva helps in the screening of Cushing's syndrome and Addisonian patients. Because sodium and potassium concentration is very well affected by corticosteroids, especially aldosterone, due to their impact on the $\mathrm{Na} / \mathrm{ATP} a \mathrm{se}$ in the striated duct cells ${ }^{13}$

\section{Stress and depressive Disorders}

Increased salivary control levels can be used as an indicator of stress. Neuroendocrine profiles were obtained for subjects experiencing military survival training using saliva sample collected at baseline and at four subsequent stress points. Cortisol levels increased significantly during the captivity experience and peaked following interrogation. Testosterone levels were significantly reduced within twelve hours of captivity. Patients with affective disorders secrete significantly less saliva than normal. Salivary flow rate is reduced in patients with clinical depression more often because of psychoactive drugs that the disease per se. In the saliva of patients with major depressive disorders, the concentrations of immunoreactive prostaglandins are significantly higher than those of healthy controls ${ }^{14}$.

\section{Saliva and cancer}

Navarro et al (1997) demonstrated that EGF concentrations were higher in the saliva of women with primary breast cancer or a recurrence of breast cancer when compared with women without disease. Boyle et al (1994) showed the value of p53 in saliva as a marker for squamous cell carcinoma, detected and identified tumor-specific mutations in p53 in preoperative salivary samples of individuals suffering from head and neck squamous cell carcinoma. Also, salivary kallikrein were observed in patients diagnosed with malignant tumors as compared with those individuals diagnosed with benign tumors and salivary CA 125 assay who had a better diagnostic value than the comparable serum assay 5,16,17. 


\section{Saliva and Sjogren's syndrome}

A number of studies have shown that if the disease is developing in the salivary glands, the periductal infiltrate and its products (cytokines) can have a profound effect on the resorptive, transport and synthetic function of the striated duct cells. This results in elevated sodium and chloride concentration and a decreased phosphate concentration despite reduced flow rate, elevation in lactoferrin, elevation in $\beta 2$ microglobulin and in Kallikrein. Parotid Lysozyme was found to be elevated in patients with primary Sjogren's syndrome but not in secondary Sjogren's syndrome ${ }^{18}$.

\section{Cystic Fibrosis}

In cystic fibrosis, the most dramatic changes reported have been an elevation in calcium and proteins, especially in the submandibular, sublingual and minor salivary glands ${ }^{19}$.

\section{Celiac Disease}

Celiac disease is a congenital disorder of the small intestine that involves malabsorption of gluten. Salivary IgA-AGA measurement has been reported to be a sensitive and specific test for the screening of this disease and monitoring the patient's adherence to the required gluten free $\operatorname{diet}^{20}$.

\section{Saliva in Drug monitoring}

Literature reveals that nowadays it is easy, safe, and efficient to use salivary samples to detect and monitor various drugs to aid in the detection of illicit drugs and thus to prevent drug abuse which includes drugs like cotinine, cannabinoids, cocaine, phencyclidine, opioids, barbiturates, diazepines, amphetamines, and ethanol. But, the salivary drug measurement is made difficult due to the unwanted materials in the whole saliva like bacterial products and epithelia cells which make the collected samples unstable, and these can be dealt with with the use of salivary sample preservatives and advanced/improved saliva collection methods and devices. $21,22,23,24,25,26$

\section{Saliva in Hormone monitoring}

Various steroids like estradiol, estriol, cortisol, dehydroepiandrosterone, progesterone and testosterone are nowadays assessed by salivary samples. Saliva levels of steroid hormones reflect the free/and thus active, level of these hormones. ${ }^{25,26}$

\section{Saliva in Toxicology}

Over the counter and prescription, drugs and the so called "recreational drugs" like tobacco, alcohol, cocaine and marijuana can be detected and measured using saliva. Recreational drugs monitored in saliva include marijuana theophylline, cocaine, caffeine and alcohol. FDA approved orasure oral specimen collection system claims to be a sensitive and specific kit to detect any of the NIDA 5 drugs that is cannabinoids (marijuana), cocaine, methamphetamine, opiates and phencyclidine hydrogen chloride using a single Orasure specimen ${ }^{25,26}$.

\section{Saliva in Forensic sciences}

Salivary samples obtained from various sites like the victim's fingernails, suspect's blood, semen or scratched skin, contain DNA which is characteristic for different individuals, and salivary DNA analysis is used in forensic medicine and dentistry and is now frequently used in identifying individuals ${ }^{26}$.

\section{Barriers in salivary Diagnostics}

There are 3 basic types of barriers to salivary diagnostic techniques. ${ }^{27}$

The first is associated with research which requires a long term investment which is not possible for all private and government sectors.

The second deals with financial issues or the cost of product where cooperation by both private and government agencies is needed.

Finally, with third party acceptance and associated legal issues and to overcome this medical insurance companies have to 
be convinced about cost-effectiveness and accuracy of salivary tests.

\section{Nano-Diagnosis: Future of Salivary Diagnosis}

With the emergence of nanotechnology, Oral Fluid NanoSensor Test (OFNASET) is gaining attention as it is easy-to-use and handheld. It helps in the simultaneous and rapid detection of multiple salivary protein and nucleic acid targets. Even it can be used in dental office for the screening of diseases. Clinical applications include oral cancer, breast cancer and metabolic diseases say type 2 diabetes. ${ }^{28}$

\section{Conclusion}

Saliva as a diagnostic tool is giving promising results in the diagnosis of infections, cardiac diseases, oral cancer, psychiatric disorders, drug monitoring, nephrology, dental caries, auto-immune disorders and many more. Latest advances in technology have made use of saliva as a valuable, non invasive diagnostic aid i.e. to study microbes, chemicals, hormones and immunological markers. At present, saliva is not only used for the diagnosis and monitoring of oral diseases, but it serves a very important role in the diagnosis and monitoring of various systemic diseases. Many areas of research like nanotechnology involving salivary components and functions are in progress for local and systemic disease diagnosis, treatment and prevention. Dentistry should support the development and promotion of saliva as a diagnostic fluid for detecting, preventing and monitoring of the overall health.

\section{References}

1. Rai B et al. Saliva as a Diagnostic Tool in Medical Science: a Review Study. Advances in Medical and Dental Sciences 2008; 2(1): 9-12.

2. Gopinath VK, Arzreanne AR. Saliva as a Diagnostic Tool for Assessment of Dental Caries. Arch Orofac Sci 2006; 1: 57-9.
3. Leone CW, Oppenheim FG. Physical and chemical aspects of saliva as indicators of risk for Dental caries in Human. J Dent Educ 2001; 65:1054-64.

4. Sawasdivorn S, Wanthanaphuti P, Puearun S, Juansang S. Situation of baby bottle use: is it suitable to recommend weaning by the age of one year? J Med Assoc Thai 2008; 91 Suppl 3: S128-35.

5. Driessens FCM, Borggreven JMPM, Verbeeck RMH, J.W.E van Dijk F.F Feagin. On the Physicochemistry of plaque calcification and the phase composition of dental calculus. J Periodontol Res 20(3): 329-36.

6. Kudva P, Tabasum ST, Sharma S, Gupta S. Role of Saliva as a Diagnostic Tool in Periodontal Disease. Arch Dent Sci 2010; 1(1): 21-5.

7. Kaufman E, Lamster IB. The diagnostic applications of saliva- a review. Crit Rev Oral Biol Med 2002; 13(2):197-212.

8. Llena-Puy C. The role of saliva in maintaining oral health and as an aid to diagnosis. Med Oral Patol Oral Cir Bucal 2006; 11: E449-55.

9. Lamey PJ, Nolan A. Anti HIV antibody in saliva: an assessment of the role of the component of saliva, testing methodologies and collection systems. J Oral Path Oral Med 1996; 25: 104- 7.

10. Robinson JL, Lee BE, Kothapalli S, Craig WR, Fox JD. Use of throat swab or saliva specimens for detection of respiratory viruses in children. Clin Infect Dis 2008; 46: e61-4.

11. Amer $S$ et al. Salivary glucose concentrations in patients with diabetes mellitus - a minimally invasive technique for monitoring blood glucose levels. Pak J Pharmaceut Sci 2001; 14(1): 33-7.

12. Denver. Salivary endothelin concentrations in the assessment of chronic heart failure.

13. The Lancet 2000; 355: 468-9. 
14. Raff H. Utility of salivary cortisol measurements in cushing's syndrome and adrenal insufficiency. J Clin Endocrinol Metab 2009; 94(10): 3647-55.

15. Marita Pruessner et al. Self-Reported Depressive Symptoms and Stress Levels in Healthy Young Men: Associations with the Cortisol Response to Awakening. Psychosomatic Medicine 2003; 65: 92-9.

16. Boyle JO, Mao LE. Gene mutations in saliva as molecular markers for head and neck squamous cell carcinomas. Am J Surg 1994; 168: 429-32.

17. Hu S et al. Salivary proteomics for oral cancer biomarker discovery. Clin Cancer Res 2008; 14(19): 6246-52.

18. Navarro MA et al. Epidermal growth factor in plasma and saliva of patients with active breast cancer and breast cancer patients in follow-up compared with healthy women. Breast Cancer Res Treat 1997; 42: 83-6.

19. Sreebny LM, Zhu WX. The use of whole saliva in the differential diagnosis of Sjoe gren's syndrome. Adv Dent Res 1996; 10: 17-24.

20. Lawson D, Westcombe P, Saggers B. Pilot Trial of an Infant Screening Programme for Cystic Fibrosis: Measurement of Parotid Salivary Sodium at 4 months. Arch Dis Childh 1969; 44: 715.

21. Pastore L, Campisi G, Compilato D, Muzio LL. Orally Based Diagnosis of Celiac Disease: Current Perspectives. J Dent Res 2008; 87(12): 1100-7.
22. Schramm $W$ et al. Cotinine in an ultrafiltrate of saliva. Prev Med (1992 b) 21;63-73.

23. Schramm $W$ et al. Drugs of abuse in saliva; A reveiew. J Anal Tox (1992 c) 16; 19.

24. Schramm $W$ et al. Methods of simplified saliva collection for the measurement of drugs of abuse, therapeutic drugs and other molecules. Ann NY Acad Sci (1993) 694;311-313.

25. Smolle et al KH. Q.E.D alcohol test- a simple and quick method to detect ethanol in saliva of patients in emergency department. Comparison with the conventional determination in blood. Intensive Care Med (1999) 25;492-495.

26. Chiappin S, Antonelli G, Gatti R, De Palo EF. Saliva specimen: A new laboratory tool for diagnostic and basic investigation. Clinica Chimica Acta 2007; 383: 30-40.

27. Lima DP, Diniz DG, Moimaz SAD, Sumida DH, Okamoto AC. Saliva: reflection of the body. Int J Infect Dis 2010; 14: e184e8.

28. Christopher H. Saliva as a Diagnostic and Monitoring Tool. American Association for Dental Research, October (2009); w w w. de n talresearch.org.

29. Wong DT. Salivary diagnostics powered by nanotechnologies, proteomics and genomics. J Am Dent Assoc 2006; 137: 313-21. 